\title{
Federico García Lorca e Rafael Alberti: I'amicizia amputata. Cronaca di un percorso unitario per la difesa dei diritti civili: Alberti e gli epicedi per il "fratello" andaluso
}

\section{Lorenzo Spurio}

Critico letterario e poeta, Presidente dell'Associazione Culturale Euterpe di Jesi

https://doi.org/10.33676/EMUI nomads.59.11

\section{ALCUNI CENNI BIOGRAFICI SU RAFAEL ALBERTI}

Rafael Alberti nacque a Puerto de Santa Maria (Cadice) nel 1902 (tra i suoi avi, i nonni erano di origine italiana) e morì nel suo stesso paese natale nel 1999. Poeta appartenente alla generazione del ' $27^{1}$, fu poeta impegnato (poeta comprometido) schierato pubblicamente nelle file della sinistra. Esponente centrale della causa rivoluzionaria, fondò le riviste Octubre e Revista de los artistas revolucionarios, divenne poi direttore della rivista El mono azul, molto popolare tra le fine repubblicane. Con l'avvento della guerra civile decise di esiliarsi e visse per circa vent'anni in Argentina e a seguire per quattordici anni a Roma. A dittatura collassata, fece ritorno nel suo paese. Tra le principali opere di Alberti vanno citate Marinero en tierra (1924), Cal y canto (1929), Entre el clavel y la espada (1941), Retornos de lo vivo lejano (1952). Tradotto in italiano dall'amico spagnolista Vittorio Bodini che nel 1963 lo inserì nell'antologia I poeti surrealisti spagnoli, opera nella quale aveva contemplato anche i poeti Gerardo Diego, Federico García Lorca, Vicente Aleixandre, Juan Larrea, Luis Cernuda, Manuel Altolaguirre, José Moreno Villa ed Emilio Prados. Appartiene alla cosiddetta fase surrealista di Alberti l'opera Sobre los ángeles (1929) ${ }^{2}$ che Ignazio Delogu definì un'opera non in grado di celare una «profonda e paurosa crisi personale» dell'autore ${ }^{3}$. Inizialmente critico verso la sua produzione fu 
Carlo Bo che nelle sue note Carte spagnole (1948), come ricorda la critica Maira Negroni, «sembra non apprezzare l'estrema varietà di temi e di registri [...] che secondo lui parrebbe nata più dal virtuosismo del poeta che da autentiche esigenze interiori di espressione ${ }^{4} \gg$. La grande diffusione di Alberti in Italia si deve senz'altro alle edizioni curate e allo strenuo impegno di critici come Vittorio Bodini, Oreste Macrì e Ignazio Delogu ${ }^{5}$. Alberti fu in contatto con Carlo Levi, Giuseppe Ungaretti, Pier Paolo Pasolini e Márcia Theóphilo (solo per citarne alcuni).

Pure non va minimamente trascurata l'intensa attività pittorica di Alberti influenzata di certo da Picasso - che, se viene considerata come secondaria rispetto a quella letteraria, ebbe degli esiti rimarchevoli al punto tale che critici di spessore se ne occuparono. Si dedicò all'arte dell'incisione ma non solo; una versione dei $X$ sonetos romanos in edizione limitata uscì nel 1964 accompagnata da sue acqueforti e incisioni a piombo. Carlo Bo rintracciò in questa sintonia di parola e immagine (lui stesso le definì con l'uso di un avvincente neologismo liricografie) una fortunata filiazione diretta ai Calligrammes di Apollinaire ${ }^{6}$. Maira Negroni sostiene che «Attraverso la pittura e l'incisione, la grafica e le liricografie, l'illustrazione di libri e il commento poetico d'arte, Alberti dimostrò di poter essere riconosciuto, oltre che come poeta, anche come artista di talento ${ }^{7}$. Per conoscere in maniera più attenta le fasi che contraddistinsero la sua esistenza rimane utilissima la sua autobiografia in vari volumi La arboleda perdida (1959), aggiornata in vari fasi della sua vita. Ignazio Delogu ha osservato che «La vittoria e la durata della dittatura franchista hanno poi fatto sì che per lunghi anni non solo l'opera di Alberti, dalla poesia al teatro, fosse proscritta in Spagna, ma che persino il suo nome venisse taciuto nelle antologie e nelle storie letterarie o ridotto a una citazione puramente nominale ${ }^{8}$ ». Medesimo trattamento ebbero tanti altri poeti quali Luís Cernuda, Federico García Lorca (sebbene assassinato, la sua opera venne proibita, in stampa e diffusione, sul territorio spagnolo), Miguel Hernández e molti altri.

Per completare un affresco minimo della figura di Rafael Alberti va subito detto del suo impegno ideologico e politico che lo vide promotore e sostenitore 
di battaglie, partecipante di manifestazioni, firmatario di manifesti e sostenitore di cause internazionali in difesa del popolo, degli oppressi e dei dimenticati, mai venendo meno alla sua lotta per la conquista della democrazia e della libertà. Aspetti che ritroviamo nella sua opera, tanto poetica che teatrale, per il quale viene legittimamente ricordato come poeta sovversivo, el poeta en la calle, poeta comunista, marxista e rivoluzionario. Alcuni accenni a questo suo impegno vanno dunque riportati.

Fervente anticlericale e antimonarchico, nel 1932 viaggiò in Unione Sovietica e prese parte al Primo Congresso Mondiale contro la guerra, presiedúto da Henri Barbusse. Due anni più tardi partecipò al Primo Congresso degli scrittori Sovietici. Ritornato in Spagna, all'inizio del 1936, partecipò alle attività di protesta sostenendo il Frente Popular. Fu sempre in prima linea e molte furono le sue presenze - assieme alla sua compagna María Teresa León (1903-1988) - a iniziative di propaganda e sostegno della Repubblica, indossando la casacca comunista. Dal temperamento battagliero fu lui stesso a ricordare di aver fatto allontanare con il solo uso della parola, in un convegno presso l'Ateneo di Cadice dove era stato invitato, il poeta José Maria Pemán ${ }^{9}$ (falangista, futuro poeta di regime nonché Presidente della Reale Accademia della Lingua Franchista). Ebbe a ricordare dell'avvenimento: «Era la prima volta che lo vedevo. Le uniche parole che io gli abbia mai rivolto nella vita furono: Faccia il favore di abbandonare la sala». Alberti-Pemán, una delle metafore piú palesi e radicate delle due Spagne, che, pur non potendo mai riavvicinarsi del tutto - complice un passato pesante che non va dimenticato - si ritrovarono a dittatura ultimata, in un incontro a Cadice (la loro comune città) nel 1981, poco prima che Pemán morisse. Momento significativo immortalato da uno scatto in bianco e nero riportato dalla stampa del tempo, contornato da una cronaca di distensione dove, appunto, si alludeva all'incontro delle due Spagne: «Ninguna historia tan cierta como la que ocurrió allí, en la plaza de San Antonio, la tarde que Rafael Alberti pronunció el pregón del Carnaval gaditano, no disfrazado, sino vestido del uniforme del cuerpo al que pertenece, pues iba el poeta ataviado de Marinero en Tierra. La familia, que sabía de las debilidades liberales de 
Pemán, le había recriminado: "Papá, no se te vaya a ocurrir por nada del mundo salir a saludar a Alberti, que te conocemos..." Estaba Pemán ya enfermo y torpón, y cuando oyó el estruendo carnavalero del pregón de Alberti, ni corto ni perezoso salió a la puerta de la casa, no para ver el cadáver del enemigo, sino para abrazar al amigo. Se abrazaron los dos. Pemán dijo a Rafael: "Como poeta, Rafael, no hay color..."Y no había colores, ni morados ni gualdos, en aquel abrazo con un fondo de papelillos y serpentinas. Los pitos de caña tuvieron que sonar a la concordia de la marcha granadera. Porque aquella tarde, me ha contado la luz del faro de la Caleta, terminaron en Cádiz las dos Españas ${ }^{10} »$.

Dopo i vari periodi di esilio (Argentina e Italia) e una volta spazzata via la dittatura franchista, Alberti ritornò nel suo paese dove nel 1977, quale coronamento di un'intera vita spesa per la difesa dei diritti, venne eletto, nelle file del Partito Comunista, al Parlamento nel collegio della sua zona, Cadice, nel mese di giugno. Tuttavia, pochi mesi dopo (settembre) presentò le sue dimissioni dall'incarico alle Cortes, lasciando il suo seggio a un dirigente dei contadini andalusi. Uno dei molteplici gesti di umanità e coerenza, nonché di onestà intellettuale, di cui la sua lunga vita risulta contrassegnata.

\section{L'ESILIO ITALIANO}

Parlando di Rafael Alberti, in questa circostanza, alcuni cenni al periodo dell'esilio trascorso a Roma sembrano necessari. Un approfondimento di tale importante fase creativa e d'impegno del poeta spagnolo è reso dalla tesi di laurea di Maira Negroni, poi pubblicata in volume con titolo Rafael Alberti: l'esilio italiano (2000). La studiosa va rintracciando con meticolosità i temi, le influenze e le suggestioni percepite da Alberti a Roma nel periodo 1963-1977 ravvisabile nella produzione di due libri tra loro particolarmente differenti, eppure scritti in un periodo coevo. Le due opere in questione sono Roma, peligro para caminantes (1964-1967) e Canciones del Alto Valle del Aniene (1972). Nel primo ${ }^{11}$ troviamo composizioni che affrescano la Roma del momento: non tanto quella imperiale e gloriosa, ma la Roma suburbana, caotica e inflessibile a ogni 
romanticismo, dominata da una soffocante spersonalizzazione, sporcizia e precarietà. Roma non è tanto, in questi componimenti, il centro della tradizione e della grandezza del passato ma un luogo quasi ostile e pericoloso. Si tenga presente il titolo stesso del libro, anomalo se si considera che è una raccolta di poesie, e questo è così troppo tecnico, descrittivo, atto a decifrare in maniera chirurgica uno strato della realtà. La Capitale, pur nel suo indubbio fascino stilistico-architettonico, è luogo di pericolo ${ }^{12}$ anche per lo spensierato camminante. Ed è così che in questo volume, strutturato in cinque sezioni, come un percorso vero, condotto a piedi, per quartieri o zone intersecanti, ci si "sofferma sulla Roma viva, chiassosa, prosaica di Trastevere e di via Garibaldi ${ }^{13}$ ». Ciò comporta l'utilizzo di un linguaggio franco e spietato, finanche aggressivo con intercalari locali e coloriture espressive, con la finalità di rendere quell'oralità tra il popolare e il becero alla quale assiste, un po' frustrato, un po' affascinato. Assistiamo a pagine ben caratterizzate, testimoni di un realismo crudo e impietoso, anche il linguaggio si abbassa ${ }^{14}$ - rispetto alle sue prime produzioni enfatiche e rigogliose - al gergo della strada. Sono state ravvisate pennellate, seppur recondite, di ironia in uno scenario che è prevalentemente ambiguo e apparentemente infido: la degradazione prodotta col verso sembra in alcuni estratti addirittura peggiorativa a voler sottolineare, forse, con un anticlimax il fastidio che prova dinanzi alla visione del delirio e della confusione generalizzata. I versi parlano della banalità e del controsenso della vita abituale dei quartieri popolari. Traffico, caos, crepe sui muri dei palazzi ${ }^{15}$, immondizia e volgarità della gente, vicoli infestati dai gatti ${ }^{16}$, un senso di pervasiva claustrofobia e l'attenzione per la dimensione scatologica dell'uomo (soprattutto l'atto dell'orinare ${ }^{17}$ ) fanno da padroni in questa denuncia al consumismo dove pure si ravvisa una banalizzazione del sacro e un veemente anticlericalismo con riferimenti a sedicenti preti che fanno contrabbando o macchiati da peccati capitali. Meritano un'attenzione particolare i Nocturnos qui contenuti, componimenti dove l'aspra visuale del contesto ambientale sembra leggermente smorzata per dar risalto a una riflessione interiore. Con il calare delle luci naturali e della ribalta del mondo egocentrico, il poeta sembra ricollegarsi a una 
dimensione più intima e dolce, finanche dai tratti filosofici. Negroni ha osservato che «il fascino misterioso della città notturna si connota di una sorta di presagio mortale» (51). In questo libro Roma inghiotte il poeta: Alberti sente l'asfissia farsi pressante, come se una grande bocca lo fagociti un po' alla volta, mentre quell'ambiente corrotto scorre sotto ai suoi occhi, senza lasciargli scampo.

Le opere contenute in Canciones del Alto Valle del Aniene $(1972)^{18}$ sono scritte nella ricerca di un luogo amico e pacificato, lontano dal trambusto della megalopoli, nella sua casa del piccolo borgo di Anticoli Corrado, sui Colli Romani. Scritto nel periodo del duro conflitto internazionale per il destino del Vietnam, Alberti si sente profondamente infastidito dalle violenze perpetuate dall'aggressore a stelle e strisce ma con questa opera "cerca di sospendere la sua dolorosa coscienza della guerra e della morte concentrandosi nella contemplazione del paesaggio ${ }^{19}$ ". Ecco perché le poesie qui inserite sembrano aver dimenticato l'opacità e la convulsione della Roma nevralgica, per permettere al poeta di trovare quella solitudine che va ricercando. Sono «poesie della comunione con la natura ${ }^{20}$ ", come le ha definite Negroni, dove il poeta va rintracciando i valori e i significati nella semplicità delle immagini, nella natura, nel collante popolare che si esplica nella condivisione sincera, nei momenti di festività come la celebrazione del patrono. II poeta, che riesce ad allinearsi a un sentimento di empatia con l'ambiente e di rilassamento generalizzato, ha mutato anche il tono per riferirsi al lettore: più disteso, quasi estasiato dinanzi alla bellezza del semplice, in una dimensione di ricerca introspettiva e di progressivo completamento. In quel contesto e con un animo risollevato dalle bassezze quotidiane della Roma spasmodica, il poeta è in grado di distanziarsi da quel malumore costitutivo della sua poesia civile, di quell'inquietudine di fondo che lo pervadeva e che lo induceva a declamare con stizza e inclemenza verso le ingiustizie e i poteri forti.

\section{ALBERTI E GARCÍA LORCA: UN'AMICIZIA SENZA FINE}

Rafael Alberti e Federico García Lorca s'incontrarono per la prima volta, 
grazie al poeta malaghegno José Moreno Villa (1887-1955), nell'ottobre del 1924 a Madrid presso la Residencia de Estudiantes ${ }^{21}$, quel centro nevralgico di incontri ed esperienze culturali che rappresentò per la Spagna e non solo una delle fucine più intraprendenti e ricche della cultura contemporanea. Lì transitarono, tra gli altri, medici, filosofi, scienziati di chiara fama mondiale e si aprirono una serie di collaborazioni tra artisti, alcuni dei quali strinsero una vera e propria amicizia che sarebbe durata nel tempo, anche venuta meno l'esperienza della "Resi". Tra di loro vanno senz'altro ricordati, per quanto è di nostra attenzione nel presente saggio, Rafael Alberti, Luís Cernuda, Pedro Salinas, Gerardo Diego, Federico García Lorca nonché il pittore Salvador Dalí, il regista Luis Buñuel e tanti altri ancona. Maria Cristina Assumma riporta che nel loro primo incontro - che dovette essere stato davvero "catartico", come avviene forse solo tra amanti - García Lorca recitò quello che sarebbe nel tempo diventato il celebre "Romance sonámbulo". In cambio Alberti, che ancora non era approdato completamente e con gli esiti che oggi conosciamo, alla poesia ed essendo in quella fase più legato alla pittura, gli fece dono di un quadro. Nel corso del tempo scambi e omaggi di questo tipo non vennero mai meno.

Uno di questi è l'opera tetrapartita di Alberti dal titolo "A Federico García Lorca poeta de Granada" datata 1924 composta da quattro sonetti stagionali22 che sarebbero poi apparsi in Marinero en tierra, tranne "Verano", che rimase inedito e che Federico probabilmente non lesse mai ${ }^{23}$. Nei quattro componimenti fuoriesce distintamente la neonata amicizia dei due, la profonda stima di Alberti per Federico, il dulce amigo ${ }^{24}$, che riconosce quale voce autentica della poesia popolare, sentimenti dei quali non solo Federico dovette essere entusiasta ma anche profondamente partecipe dedicandogli a sua volta alcune poesie. Con evidenza Alberti ben conosceva le suggestioni visive e le simbologie care a Federico - del quale aveva ascoltato, forse, recitare qualche suo testo che sarebbe poi finito nel Romancero gitano - come si percepisce da numerose immagini collegate alla morte, nucleo concettuale fondamentale nelle liriche del granadino - Alberti evoca il puñal, la espada e fa uso delle rispettive unità predicative con i verbi acuchillar e clavar, ma impiega anche termini quali 
cadáver, sangre -. Di Federico, Alberti scolpisce il «rostro oscuro de perfil gitano $^{25}$ », definendolo «ciervo del agua ${ }^{26}$ » e poi, ancora, in un apicale encomio naturalistico, «ciervo de espuma, rey del monterío ${ }^{27}$ », mentre egli stesso si identifica in un «corzo del mar de agua caliente ${ }^{28}$ », in cui il capriolo è chiaramente inferiore al cervo ${ }^{29}$. Centrale in questi quattro componimenti è l'isotopia dell'universo ittico, impiegata in maniera translitterata e analogica a identificare Federico: in "Otoño" si parla di alfanjes del río (pesce spada) e in "Verano" di pez-espadas, della cui punta perigliosa il poeta anela l'infilzamento quasi per conseguire un'iniezione di sapienza; l'abbiamo già detto, lo stima a tal punto da crederlo a lui maggiore.

1 due poeti andalusi furono grandi amici e lo sarebbero rimasti, non solo fino alla morte di uno dei due, ma per sempre. Lo dimostrano in maniera nitida i versi che, nel tempo, si scambiarono, omaggiandosi poesie, il clima di profonda stima e rispetto che li accomunava, come veri fratelli, la sana condivisione artistica, di cultura illuminata, che li portava ad essere felici per i successi dell'altro. Cosa che, oggi come allora, era assai rara. La loro comune anima andalusa, di uomini del sud impregnato di gitanismo e di gusto popolare, nonché il radicamento stretto con la terra nei suoi sortilegi fascinosi, nelle brezze marine di Malaga in Alberti e dei campi vergati dai torrenti inLorca, li avevano in qualche modo resi affini geneticamente, ben prima di un loro effettivo incontro. Tuttavia, come ebbe a dire Bodini «Se paragoniamo la produzione [di Alberti] di tipo popolare con quella dello stesso tipo di Lorca, vediamo che mentre Federico ne accentua gli elementi irrazionali, ottenendo da tali intensificazioni dei chiaroscuri drammatici, Alberti affronta questa materia scandagliando le ombre e attirandole nella pura plasticità del segno, in una festosa combinazione che salda l'impeto della giovinezza e della grazia popolare con la raffinatezza d'un classico ${ }^{30}$ ».

Forte e duraturo il loro legame, affini i percorsi, originari della stessa terra, profondi indagatori dell'anima, immersi entrambi in uno scenario storico-politico avverso, esponenti di una pattuglia di poeti che, pur diversi tra loro, marcarono distintamente il percorso letterario, parte della critica ha messo in luce quanto sia difficoltoso parlare dell'uno senza riferirsi all'altro, accennare alla produzione 
e all'impegno dell'uno senza rievocare parallelismi tra loro, elementi di condivisione, momenti di collaborazione e quant'altro. Due poeti fratelli, per intendersi. Antonio Machado, forse unico vero vate della cultura spagnola del secolo scorso assieme a Miguel de Unamuno, come una sorta di "padre putativo" di entrambi parlò così di questi due ragazzi che osservò, seguì e sostenne nel loro percorso fino a quando la vita lo assistette (morì nel tragico anno 1936, pochi mesi dopo di Federico): «La morte di García Lorca mi ha molto rattristato. Era Federico uno dei giovani grandi poeti andalusi. L'altro è Rafael Alberti. Entrambi, a mio avviso, si completavano come espressione di due aspetti della patria andalusa: quella orientale e quella atlantica. Lorca, più carico di folklore e di campagna, era genuinamente e essenzialmente granadino. Alberti, figlio di un finis terrae, la pianura gaditana dove il paesaggio si cancella, e si accentua il profilo umano su uno sfondo di mare o di saline, è un poeta più universale, ma, a suo modo, non meno andaluso ${ }^{31}$ ».

Alla morte di García Lorca ${ }^{32}$ si inaugurò un percorso di profonda tristezza che attraversò Alberti e di cui vi è testimonianza nella sua opera biografica nonché in varie interviste. La morte dell'amico, assassinato perché partidario della sinistra, non fu mai accettata da Alberti che, a differenza di Lorca, era stato un intellettuale seriamente schierato con la Repubblica. È innegabile che Lorca fosse molto vicino - ne sentisse le esigenze, ne fosse in qualche modo voce degli emarginati e dei deboli e che solidarizzò con cause di tipo socialista ${ }^{33}$ e alcune sue opere che vennero lette come "politiche" (come è il caso del "Romance de la Guardia Civil"), ma mai si espose in maniera chiara a favore della Sinistra, mai si autodefinì comunista. II suo impegno, pur ascrivibile in quelle file (sarebbe da orbi sostenere che la sua Yerma non sia una eroina dei repubblicani!34) non venne mai da lui impiegato in maniera ideologica o con espressi intendimenti nella sua opera letteraria. A differenza di García Lorca, Alberti non solo fu poeta comprometido (definizione non così stringente, che possiamo usare anche per García Lorca, per i motivi sopra addotti) ma fu espressamente un poeta politico, un poeta comunista. La sua poesia en la calle è a difesa e denuncia di un sistema di fare le cose. Si ricordi la "Elegía Cívica"35 
con la quale nel 1930 Alberti si espresse senza particolari remore contro la dittatura di Miguel Primo de Rivera. Lui stesso ebbe a definirla una «poesia sovversiva, frutto di emozione individuale, ma che annunciava già oscuramente le strade future ${ }^{36}{ }$.

Alberti, apertamente comunista, palesemente schierato, avrebbe potuto essere - proprio per tali ragioni - il bersaglio di un'azione omicidiaria nefanda come quella che toccò all'amico, nelle campagne di Granada. E questo sentimento di colpa ${ }^{37}$, da lui particolarmente accentuato, lo accompagnò per tutta la sua esistenza, ripetendosi come un mantra, che non doveva essere Federico a cadere, ma lui. Lui che aveva alzato la voce. Lui che aveva davvero combattuto contro una fazione avversa. Lui, il comunista. L'autore di una poesia marxista e rivoluzionaria ${ }^{38}$. Non solo per la questione spagnola, dato che si batté per i diritti civili oppressi in vari luoghi del Pianeta: dal Vietnam al Cile. Maria Cristina Assumma ha osservato al riguardo: "Indubbia è l'estraneità di Lorca a una conclamata militanza politica, al cui servizio è posta, invece, l'espressione poetica albertiana, riorientata dall'intenzione finalistica propria del poeta en la calle che, infine, egli intende essere ${ }^{39}$ ».

\section{AMARI RIFLESSI DI NEW YORK}

Tra le varie esperienze che accomunarono i due può risultare utile richiamare il viaggio americano che entrambi, in periodi diversi, intrapresero e che li condusse a New York e a Cuba. Federico, in preda a una crisi amorosa che lo aveva particolarmente fiaccato (la storia con Emilio Aladrén era appena terminata) e su gentile invito dei genitori affinché si riposasse e si divertisse un po', con la sola complicità al dovere dello studio della lingua inglese, intraprese questo viaggio oltreoceano nel 1929. Soggiornò alla Columbia University e visitò la Grande Mela, trascorrendo anche dei periodi Iontani dalla frenesia della caotica New York, in Virginia soprattutto, ospite di amici. La sua permanenza, originariamente pensata per essere di poche settimane, si rivelerà una delle più lunghe, lontane dalla Spagna nel corso di tutta la sua vita. Difatti rimase in 
America per nove mesi, durante i quali, terminata l'esperienza di New York, visitò anche Cuba, prima di far ritorno in Europa. Questo viaggio, che rappresentò probabilmente la prima vera apertura di Lorca all'ambiente cosmopolita e internazionale, gli permise anche di fronteggiare alcune delle sue debolezze caratteristiche e, come fu il caso di Cuba, di professare senza inibizioni e perplessità la sua condizione sessuale. Di questa esperienza americana rimane il volume di liriche Poeta en Nueva York pubblicato solo nel 1940 da un editore messicano. Poesie aspre dove il verso si allunga e con le quali dipinge un mondo spersonalizzato, che ha eretto il denaro come suo Dio, uomini che sembrano ombre di se stessi, che vagano nelle strade vorticose della città al limitare dell'Hudson che è un fiume di petrolio, immagine di morte. II pessimismo lorchiano ben risalta da questa produzione che la critica ha inserito tra le opere del momento suggestionate dal surrealismo ${ }^{40}$. In tali poesie è ritratta un'umanità degradata, impossibilitata a comunicare, in lotta con se stessa, in perenne corsa, in un ambiente freddo e invivibile dove si consuma il peccato, si uccidono animali, si defeca e orina per la strada.

In una prosa di Rafael Alberti dal titolo "Su Poeta en Nueva York di Federico García Lorca" del $1960^{41}$, ben più di una semplice recensione al libro in oggetto, Alberti mise subito in chiaro un aspetto importante che bisogna tenere in debita considerazione, ovvero che quelle poesie dai toni disturbanti, furono scritte sì in America, ma in una circostanza di profonda solitudine. Non una solitudine in termini concreti, dal momento che Lorca - come riportano le lettere alla sua famiglia e agli amici - era sempre in compagnia di persone, intellettuali e altro genere di amicizie, ma una solitudine nativa, primigenia, che, in fondo, lo connoterà sempre: «Poeta en Nueva York deve sicuramente un buon numero di poesie alla solitudine imposta dalla non conoscenza della lingua». Ma è evidente che non sia un fatto meramente linguistico, vale a dire la comunicazione che sembra difettare di un riscontro efficace non è tanto quella Federico-alterità, ma proprio quella Federico-Federico.

Alberti, con minuzia e circostanzialità, prende in esame i vari caratteri di questa nuova poetica dell'amico partendo dal mutamento della forma (dal 
canonico ottonario a un verso liberismo fluente): «[Federico] vuol correre in libertà e su versi non legati a risonanze anteriori. Senza briglie e senza staffe, questo libro», annota. Un'opera che è - usando sempre le parole di Alberti «terribilmente triste», un «terribile libro», di un «poeta in solitudine [come se si trovasse] su un'isola castigliana assediata da un'altra lingua» nella quale, proprio come le fumose e sonnamboliche presenze dei versi, il poeta «cammina nel buio, barcolla come un ubriaco, senza sapere ciò che dice: unico modo di saperci dire la sua insonna in delirio». In una breve poesia che Alberti raccolse in Versos sueltos de cada día (1982) così ricordò l'esperienza americana di Lorca: «Por aquí Federico/ denunció el repetido/ cansancio de tus oficinas,/al triste rey de Harlem/ vestido de portero,/ aquí sufrió el delirio/ de tu poblada soledad terrible,/ lejos de su Granada».

Eppure è un'opera talmente lucida e inclemente da far credere che Federico, in quel contesto turbolento e distante dalla sua famiglia e dalla sua terra, sperimenti un nuovo modo di vedere le cose, approfondisca la sua consapevolezza, si renda edotto su aspetti esistenziali che prima non aveva concepito se non come questioni irrisolvibili. Così, se da questo murmullo interiore che genera una poesia icastica e stratificata, disorganica e figlia della società consumistica ne fuoriesce una visione alterata del mondo, fosca e irreprensibile da lui delineata con forme di ambiguità («\|l poeta si trasforma in una concentrazione di misteri, che non gli interessa capire e meno ancora decifrare», dice Alberti) in contemporanea «Federico vedeva, Federíco intravedeva, incominciava a vedere e a capire molte cose...». Quella maturazione che, forse, la permanenza a Cuba da lui vissuta come una seconda casa, verrà meglio elaborata e portata a compimento. Alberti così ci parla dell'amico andaluso sull'isola caraibica: «Quando giunge all'Avana è come se d'un tratto s'imbattesse in Cadice, in una Cadice ancor più rutilante e trasandata, più graziosa e buontempona, con quella grazia straordinaria che la elle mette nella lingua morbida e rosa dei negri cubani».

Anche Rafael Alberti visitò e soggiornò a New York (visitando gli spazi dove lo stesso Lorca era passato, come la sua camera nel residence 
universitario) e poi a Cuba, alcuni anni dopo, esattamente nel 1935 dove conobbe il poeta Nicolás Guillén. A differenza di Lorca, Alberti visitò anche il Messico vivendo quasi per un anno a Città del Messico e conoscendo gli artisti dell'arte murale: Orozco, Siquieiros e Diego Riveira.

Configurabile all'interno della cosiddetta poesia surrealista spagnola di questo periodo è anche l'opera albertiana Sobre lo ángeles (1929) con la quale, secondo Fanny Rubio, "Alberti expresa las zonas más oscuras de su psiquis» 42 dove gli angeli - ai quali è dedicato l'intero volume - «representan pasiones, pero también acciones» ${ }^{43}$. Opere come Poeta en Nueva York e Sobre los ángeles, secondo la Assumma, sono caratterizzate da una componente "trascendentalista" dal momento che esse risultano «vertebrate dalla denuncia dell'ingiustizia sociale, dalla rivolta e dall'espressione dell'angoscia» ${ }^{44}$. Ignazio Delogu, in relazione ad Alberti, aggiunge un altro importante elemento: «ll suo pensiero, anti-statunitense come lo era stato quello del García Lorca di "Huída de New York, valses hacía la civilización" concepisce la città statunitense come "una ragnatela che tesse freddamente la morte di altri popoli". [...] L'immagine di poeta en la calle che grida con forza i mali della società con cui entra in contatto, dell'Alberti del 1935, è destinata a ridimensionarsi nelle raccolte argentine scritte durante l'esilio, in cui a prevalere non è più il poeta militante, ma il poeta uomo, ferito da una condizione derivata proprio dal suo impegno civile ${ }^{45} \gg$.

Si noti la vicinanza di alcuni versi dei due poeti in riferimento alla megalopoli americana da loro visitata. Alberti in "New York (Wall Street en la niebla. Desde el Bremen)” scrive: «New York. Wall Street. Banca de sangre,/ áureo pulmón comido de gangrena,/ araña de tentáculos que hilan/fríamente la muerte de otros pueblos». La New York di Lorca, metafora del mondo capitalista e consumistico americano, è uno spazio inospitale dove «no hay mañana ni esperanza posible» ${ }^{46}$. Si assiste all'anti-paesaggismo a favore di un'immagine specchiata che ci trasmette la degradazione degli ambienti cittadini e suburbani cosmopoliti (frequente la presenza del ponte e dei vetri dei grattacieli) descritti non come scenario di riferimento, ma connotati principalmente per alcune 
attitudini degli esseri che lo abitano: si parla di paesaggi di persone che vomitano od orinano e ben si capisce quanto siamo distanti rispetto alle liriche più empatiche in relazione all'ambiente che contraddistinguono il Romancero gitano. II verso si accanisce sull'esplicitazione della materialità del mondo in cui Lorca si trova: ambienti freddi e disagevoli, strutture di gomma, nickel, la supremazia di superfici trasparenti che dovrebbero specchiare e che, invece, risultano impassibili anche al riverbero come i numerosi vetri che spesso ritornano e che, più che vetri, sembrano lamine di un metallo paradossalmente refrattario a qualsiasi tipo di conducibilità. A New York si sopravvive affranti in una lancinante "angustia imperfecta» 47 , in una metropoli vorticosa descritta con un linguaggio caro ai futuristi italiani come «el mundo de las agudas velocidades» ${ }^{48}$, antro di una solitudine metamorfizzata da nascituri e giovanissimi che si aprono le vene come avviene nella celebre "Navidad en el Hudson": "He pasado toda la noche en los andamios de los arrabales/ dejándome la sangre por la escayola de los proyectos,/ ayudando a los marineros a recoger las velas desgarradas./ Y estoy con las manos vacías en el rumor de la desembocadura.// No importa que cada minuto/ un niño nuevo agite sus ramitos de venas,/ ni que el parto de la víbora, desatado bajo las ramas,/ calme la sed de sangre de los que miran el desnudo./ Lo que importa es esto: hueco. Mundo solo. Desembocadura» ${ }^{49}$. La poesia di Lorca da tellurica si fa fangosa, fosca ed emblematica di una profonda crisi di coscienza che ha piede in ogni ambito di quel reale sovvertito. La città è insonne e la precarietà che si respira è urticante e fascinosa al contempo, impavida e massacrante e capace di aprire brecce di perplessa emotività: «Todo está roto por la noche» 50 scrive. Ancor più frammentato e tagliente, però, è il giorno che ad essa segue. Altra immagine di New York in Alberti può essere tratta dall'opera Cal y canto (1929) dove è contenuta la poesia "Telegrama"; it testo, che si caratterizza per strofe molto brevi, così recita: «Nueva York./ Un triángulo escaleno/ asesina a un cobrador.// El cobrador, de hojalata,/ y el triángulo, de prisa,/ otra vez a su pizarra.// Nick Carter no entiende nada.// ;Oh!/ Nueva York» ${ }^{51}$. Secondo Ricardo 
Senabre siamo dinanzi a un testo che pare «aproximarse a los experimentos ultraístas» 52 .

Un accenno alla cinematografia del periodo va fatto, dal momento che anche questa forma artistica entra nel grande bagaglio di fonti e influenze sulla produzione di entrambi i poeti ${ }^{53}$. Nel 1929 Alberti aveva dato alle stampe Yo era un tonto y lo que he visto me ha hecho dos tontos nel quale aveva raccolto quindici poesie dedicate ad alcuni dei maggiori comici del cinema americano, tra cui Charlie Chaplin, Harry Langdon, la coppia Stan Laurel e Oliver Hardy e Buster Keaton ${ }^{54}$. Proprio a quest'ultimo anche Federico dedicò un testo dal titolo El paeseo de Buster Keaton scritto probabilmente nel 1928. I personaggi che figurano nella storia sono lo stesso Buster Keaton, un gallo, un gufo, un negro, un'americana, una giovane e una voce. Già da questa ripartizione di personaggi - così indicati (tipi di animali, razze umane, nazionalità, età) - sembra scaturire una dimensione surreale e atipica. L'opera si apre con il verso del gallo e con l'entrata in scena di Buster Keaton accompagnato dai suoi quattro figli. II padre, di colpo, con un gesto brusco li uccide con un pugnale di legno dicendo «Poveri figliolini miei». L'azione è veramente disarmante e carica di violenza, sospesa in una atmosfera indicibile e paurosa che fa ricordare l'uccisione da parte del bravo insegnante delle varie ragazzine in La lezione (1951) di Eugène lonesco che, comunque, è un'opera di gran lunga successiva. A seguire la mattanza è il ritornello del chicchirichì del gallo mentre l'assassino si allontana su di una bicicletta. Ulteriore elemento ridicolo: piuttosto che fuggire via e nascondersi per cercare di non farsi prendere per quanto ha commesso, se ne va via quasi baldanzoso - e comunque in maniera lenta - su di una bicicletta («è piacevole andare in bicicletta» 55 , osserva, sotto un «cielo neutro» che, in realtà dovremmo immaginare rosso, di quel tanto sangue che ha fatto versare). Non solo è piacevole andare in bici, ma è addirittura «emozionante», come osserva poco dopo e tale attestazione ha veramente del comico. Difficile pensare che un uomo che, implacabilmente, ha massacrato la sua famiglia possa essere incline alle emozioni. Viene chiarito, se ancora non fosse stato evidente, che Lorca gioca con la contrapposizione del vero per tutta l'opera come quando si riferisce alla 
bicicletta di Keaton richiamandola come «l'unica imbevuta d'innocenza». Lo spazio nel quale ci troviamo è indefinito, potrebbe essere riferito a qualsiasi quartiere, città, zona, anche se poco dopo viene detto che «in lontananza si vede Filadelfia», infatti, non è poi così importante saperlo. Qui avvengono cose impossibili come il paesaggio che, molto carollianamente, «si rimpiccolisce» e altre stramberie che fanno sorridere e al contempo incutono un certo timore.

È una voce (intesa come personaggio da Lorca, prima ancora che come processo comunicativo) che interviene a riscuotere la coscienza di Buster Keaton. Gli viene detto di essere scemo e che «i suoi occhi [sono] di bambino scemo» ovvero «Che sono bruttissimi. Che sono bellissimi». C'è poila donna americana della quale Keaton osserva con particolare attenzione le scarpe pronunciando delle considerazioni assurde in quel contesto, in merito al fatto che «non dobbiamo permettere quelle scarpe [perché] ci vogliono le pelli di tre coccodrilli per farle». Altro nonsense col quale, facendo il personaggio sensibile alle questioni ambientali e animalista e proponendo questa visione come giusta perché moralmente etica, dall'altra parte fa credere che sia tacitamente consentita la violenza sugli uomini e l'infanticidio. Prima che il senso di verità (finora così manchevole) venga in qualche modo ripristinato all'interno di questa cornice assurda dove tutto è condotto in maniera irrazionale ed è incongruo a un sano ragionare (l'indicazione finale, con la quale si chiude l'opera, avverte che «Sull'orizzonte di Filadelfia brilla la stella rutilante dei poliziotti»), si realizza un altro dialogico interessante con un'imprecisata giovane la quale, semplicemente sentendo il suo nome, si terrorizza (conoscendo, forse, ciò che inizialmente è accaduto ai suoi figli). Keaton tenta di affrancarsi da quel sentimento di colpa, che in sé percepisce, ma cerca di tenerlo lontano, anche con l'impiego di ulteriori considerazioni frutto di delirio: «Vorrei essere un cigno. Ma non posso anche se vorrei. Perché, dove lascerei il mio cappello?». In questo componimento, secondo la Assumma, «Lorca costruisce il suo dialogo sfruttando [...] varie modalità espressivo-comunicative che generano una trama di intersezioni talmente fitta da obbligarci a leggere il testo a più livelli simultanei, passando da un genere all'altro: prosa, cinema, teatro, pittura, etc.»56 e ha 
parlato a ragione di ibridismo «nell'innesto su un fondo tradizionale di elaborazioni avanguardistiche» 57 .

Ignazio Delogu riporta l'attenzione sull'approccio dei due poeti nei confronti della denuncia sociale relativamente alle opere partorite dai due nel periodo 1929-1930 e pone in evidenza una differenza sostanziale: «Mentre l'impegno politico della poesia albertina nasce da quello che il poeta ha contratto con la realtà spagnola, e muovendo da quella base si congiunge al più vasto impegno di lotta internazionalista, in Lorca l'esacerbata polemica anticapitalista, scatta al contatto con l'ostile realtà degli Stati Uniti, - della quale New York appare la sintesi più disumanizzata e feroce - con tutti i condizionamenti impliciti che derivavano al poeta da una circostanziata animosità verso il gigante del Nord, colonizzatore dell'America spagnola, alla quale non era forse del tutto estraneo uno dei grandi maestri della Generazione, Ruben Darío»58.

\section{FERMÍN GALÁN E MARIANA PINEDA: IL TEATRO CIVILE}

En Madrid hay un Palacio en el Palacio un monarca, perjuro, traidor y falso.

(Fermín Galán, battute dalla Madonna)

In "Il poeta nella Spagna del 1931"59 Alberti affronta la questione della poesia civile, partendo dal grande esempio dell'americano Walt Whitman, per approfondire lo scenario socio-politico e poetico del momento nel quale si trova a scrivere. In queste pagine parla della spaccatura netta che si percepisce nell'esistenza di "due Spagne" 60 , una improntata alla difesa dell'istituto monarchico e di ideologia conservatrice e l'altra, nella quale si riconosce, pervasa da fremiti che si battono per la libertà e la democrazia e sostengono la Repubblica. Siamo nel 1931 nel periodo immediatamente successivo alla deposizione di Miguel Primo de Rivera, il dittatore, e alla dictablanda del generale Dámaso Berenguer. In queste pagine si percepisce l'animo palesemente schierato del nostro nella difesa di battaglie umane di capitale rilevanza. L'autore parla della sua opera teatrale Fermín Galán (1931), che tanto 
fece scalpore, e anche della produzione teatrale di quel periodo: «Federico García Lorca, Margarita Xirgu e Cipriano Rivas Cheríf osano fare, in piena dittatura, l'elogio della Libertà in un teatro madrileno. [...] Mariana Pineda, l'opera di Federico che fu sul punto di essere proibita, e che esalta la romantica eroina repubblicana, giustiziata a Granada, permette che il nome della Repubblica sia pronunciato qualche volta, anche se non ancora gridato»61.

Fermín Galán è l'opera di Alberti incentrata sull'amara vicenda dell'omonimo personaggio che cadde nella battaglia di Jaca difendendo le sue libertà assieme a un altro giovane, Ángel García Hernández. Le loro ceneri insanguinate - come ricorda Alberti - «avrebbero disseppellito dal panteon nel quale giaceva ormai da cinquantasette anni, il corpo della Libertà, in realtà soltanto dormiente; per agitarlo, vivo, nelle sue bandiere»62. Su questo episodio Alberti scrisse anche alcuni romances tra cui uno particolarmente irriverente nei confronti dell'istituto monarchico e del Clero, nel quale si legge: «Nelle sale del Palazzo/ cornuti ballano mazurche./ La nostra dolce Sovrana63/ gusta cognac e olive,/ e languidamente, più tardi,/ arcangelo di neve insulsa,/ tonta del fico, sviene/ su monsignor Segura. ${ }^{64}$ / Ignorato in un angoletto/ piange il Principe di Asturias $^{65}$ ».

Fermín Galán Rodríguez (1899-1930) è considerato uno dei martiri della Repubblica spagnola. Originario della provincia di Cadice, aveva preso parte alla sollevazione militare del Generale Sanjurjo nel 1926 contro la dittatura di Miguel Primo de Rivera e come condanna era stato incarcerato per sei anni (pena che sarà poi accorciata dall'amnistia concessa da Berenguer). II suo nome rimane, però, legato alla vicenda amara del sollevamento di Jaca, avvenuto nel 1930, che si rivelò come uno dei più grandi bagni di sangue, di quel periodo, tra le file repubblicane e i civili. A capo del Comité Nacional Revolucionario, assieme ad altri militari tra cui Ángel García Hernández (19001930), prese parte alla sollevazione di Jaca che iniziò il 12 dicembre 1930, con l'intenzione di dirigersi su Huesca, il maggior centro della zona. In prossimità di Cillas avvenne lo scontro tra repubblicani e le forze reazionarie del governo centrale. Secondo le testimonianze, il suo corpo sarebbe caduto a terra colpito 
dalle pallottole, mentre gridava convinto "Viva la Repubblica!".

L'omonima opera di Alberti, scritta nel 1931, venne rappresentata per la prima volta al Teatro Español di Madrid il 1 giugno ${ }^{66}$ dello stesso anno - alla presenza di Manuel Azaña - per la regia di Cipriano Rivas Cheríf, con scenografia di Sigfrido Burmann e l'attrice Margarita Xirgu quale interprete della Virgen de Cillas (oltre che della madre dell'eroe). In quanto all'estreno la stampa ricorda che non mancarono polemiche, soprattutto nel momento in cui, nel secondo atto, la Madonna entra in scena dichiarandosi di fede repubblicana e chiedendo la testa del re. Accusata di blasfemia, come pure lo stesso autore che la produsse, l'opera creò malcontento generalizzato, inserendosi in quel clima teso che avrebbe alimentato la spaccatura insanabile tra le due Spagne. La Madonna entra in scena, irriguardosamente, abbracciando una baionetta e intervenendo in soccorso dei cospiratori che si sono sollevati, impiegando un tono iroso e belligerante, incitando alla lotta. Queste sono alcune delle parole che tanto fecero inalberare le numerose schiere di monarchici presenti in sala: «Yo defiendo la República/ y a los revolucionarios./ ¡Abajo la monarquía!// Salid conmigo a los campos./ ¡Dadme un fusil o un revólver,/ una espada o un caballo!// Quiero ser la coronela/ de todos los sublevados./ ;Seguidme, amigos, seguidme!». Attacchi mirati al clero da parte di Alberti con questa opera non mancarono e furono diretti al Cardinale Segura. Racconta Alberti in La arboleda perdida: «el teatro entero protestó violentemente: los republicanos ateos porque nada querían con la Virgen, y los monárquicos por parecerles intolerables tan criminales sentimientos en aquella Madre de Dios que yo me había inventado. Pero lo peor faltaba todavía: el cuadro del cardenal monseñor Segura, borracho y soltando latinajos molierescos en medio de una fiesta en el palacio de los duques»67. I rappresentanti della destra ivi presenti (non solo i monarchici ma anche appartenenti alla destra repubblicana), sfiduciati, inveirono contro gli attori e l'autore dell'opera al punto tale che venne fatto calare dall'alto il telone. Solo una volta abbandonato il teatro, per i rimasti, la rappresentazione poté continuare fino all'epilogo scritto da Alberti con la madre che a Fermín Galán recita addolorata ma onorata: «Tu sangre por la República/ ya dio sus banderas 
granas./ Tuviste tú que morir/ para libertar a España».

Felicemente, con l'avvento della Seconda Repubblica, la ritrosia e la censura imposta a numerosi intellettuali che erano stati profondamente ostacolati, repressi e che avevano visto negato il diritto di rappresentare le loro opere, viene a mancare. Di questa nuova fase, che fa ben sperare, ne parla Alberti: «Gli intellettuali erano felici. Ormai è possibile rappresentare le opere di teatro proibite. La farsa de la reina castiza, di Valle-Inclán, viene rappresentata da Lopez Heredia [...] L'attrice repubblicana, la vera amica dei poeti e degli scrittori, Margarita Xirgu, rappresenta La corona di [Manuel] Azaña, e il mio Fermín Galán. [...] Anche García Lorca ha un'opera proibita: Amor de Don Perlimplin con Belinda en su jardin, che non poté essere rappresentata perché ritenuta immorale, secondo la dittatura». Rientra perfettamente in questo clima l'opera Mariana Pineda (1927) di Lorca sebbene la battaglia della sua eroina non sia collocabile nelle ostilità repubblicani-conservatori che avrebbe condotto alla fondazione della Seconda Repubblica, ma sia precedente. Difatti essa è calata in uno scenario storico-politico antecedente e la sua battaglia liberale è condotta contro il dispotismo di Re Ferdinando VII di Borbone (1784-1833). Tenendo presente questa precisazione, va comunque affermato che la battaglia condotta da Mariana è una battaglia per la libertà e la democrazia, volta all'abbattimento dell'istituto monarchico, sentito come vetusto, lontano dalla esigenze della gente e accentratore, al punto tale che, durante gli anni 19301931, ella venne assunta come uno degli emblemi dei repubblicani. Diversi i periodi storici, dunque, ma stessi gli ideali.

La bella popolana Mariana Pineda y Muñoz (1804-1831) si era sposata con Manuel de Peralta y Valle, uomo undici anni più grande di lei, che stava in procinto di ritirarsi dall'esercito e dall'unione erano nati due figli. Nel 1822 Manuel de Peralta morì lasciandola sola a crescere i figli. In concomitanza con il fervore liberale sfociato contro l'assolutismo di Re Ferdinando VII, Mariana simpatizzò e poi prese parte in maniera entusiastica alla causa liberale, rendendosi anche disponibile a ospitare nella sua casa alcuni perseguitati come Casimiro Brodett y Carbonell. In questa sua attività di aiuto e sostegno nei 
confronti dei cospiratori, Mariana decise di aiutare un suo cugino, Fernando Álvarez Sotomayor, che era stato condannato alla prigionia per aver partecipato a manifestazioni avverse al regime. Con uno stratagemma riuscì a farlo uscire di prigione e si adoperò per metterlo in salvo mediante dei documenti falsi per andare all'estero. Parallelamente, durante il periodo nel quale lei lo nascose nella sua casa, nacque tra i due una storia d'amore che sarà il principale nutrimento di Mariana nei confronti della difesa dell'uomo e del sostegno della causa liberale. Lalcalde del crimen Pedrosa, una volta che Sotomayor si fu dileguato dalla casa, cominciò a tenerla sott'occhio con interrogatori e perquisizioni ma lei, per l'amore che la legava all'uomo, mai rivelerà di averlo aiutato e nascosto nella sua casa né dove era riparato. Così venne arrestata perché le forze governative trovarono nella sua casa una bandiera da lei cucita con colori e simbologie rivoluzionarie $e$ in seguito venne rinchiusa in un convento. Le venne poi promesso un indulto che l'avrebbe risparmiata dalla morte, se avesse rivelato dettagli utili in merito a Sotomayor ma lei non assecondò mai le richieste dei suoi aguzzini e all'età di ventisei anni venne assassinata. Lo sfegatato liberalismo della donna, motivato sia dall'insofferenza nei confronti di re Ferdinando VII (qui rappresentato da Pedrosa) quanto dal desiderio di ricongiungersi al suo amato, fecero di lei una persona nella quale risulta difficile separare la sfera privata (l'amore, il sentimento e il desiderio di combattere per l'ottenimento del suo uomo) da quella pubblica (la lotta contro la tirannia e la fondazione di uno stato che rispetti i diritti di tutti). La ribellione di Mariana risiede in un agognato sistema politico ugualitario, liberale; l'annullamento di forme e gerarchie di potere secondo Mariana Pineda potrà realizzarsi solamente con il perseguimento di un piano insurrezionale (una cospirazione) che utilizzerà dunque la forza per deporre i despoti e aprire al nuovo, al rispetto delle libertà umane. Così recita nell'opera, assediata dalle domande di Pedrosa e già incamminata verso la tragica fine che l'aspetta: «Amas la libertad por encima de todo,/ pero yo soy la misma Libertad. Doy mi sangre,/ que es tu sangre y la sangre de todas las criaturas./ ¡No sé podrá comprar el corazón de nadie!/ [...]/ ¡Yo soy la Libertad porque el amor lo quiso!/ ¡Pedro! La 
Libertad, por la cual me dejaste./ Yo soy la Libertad, herida por los hombres!». L'opera, alla quale Lorca aveva lavorato nel periodo 1923-1925, venne pubblicata nel 1927 e rappresentata al Teatro Goya di Barcellona il 24 aprile del medesimo anno dalla compagnia di Margarita Xirgu (nel ruolo principale) con preziose scenografie di Salvador Dalí. Sarebbe poi stata ripresentata pochi mesi dopo (il 12 ottobre) al Teatro Fontalba di Madrid.

\section{SCAMBI POETICI ED EPICEDI PER FEDERICO}

En Víznar

el agua me espera para llorar.

\section{A Víznar}

quizá llegue un día para no llorar

(RAFAEL ALBERTI, 1978)

Numerosi i testi che nel corso della sua lunga vita Rafael Alberti dedicò all'amico García Lorca. Per alcuni di essi è diretto il riferimento al dedicatario, per altri, invece, Federico non viene citato espressamente ma è facile intuire che siano a lui dedicati. Nella poesia "Retornos de un poeta asesinato" contenuta in Retornos de lo vivo lejano, che raccoglie opere del periodo 1948-1956, si legge di un «abrazo mudo que me has dado» che non facciamo difficoltà a immaginare sia quello dell'amico ormai assente, di cui sente la presenza (l'abbraccio) anche se esso è svuotato di brio ed energia (è muto).

In "Balada del andaluz perdido" contenuta in Baladas y canciones del Paraná che contiene poesie del periodo 1953-1954, sebbene il poeta si trovi nel continente sudamericano, è evidente quanto il pensiero ricorrente - quasi una vera ossessione, com'è stato rivelato dalla stessa figlia di Alberti - per Federico sia totalizzante: «Vería el odio, la guerra,/ cerca tal vez de otro río»; in "Balada del que nunca fue a Granada" leggiamo: "Venid los que nunca fuisteis a Granada./ Hay sangre caída, sangre que me llama./ Nunca entré en Granada.// Hay sangre caída del mejor hermano./Sangre por los mirtos y aguas de los patios./ Nunca fui a Granada.// Del mejor amigo, poir las arrayanese./ Sangre 
por el Dauro, por el Genil sangre./ Nunca vi Granada». Inconcepibile visitare Granada, la sua città e la sua zona, senza pensare di non poter avvertire la presenza umana di Federico che Alberti qui definisce il mejor hermano e il mejor amigo. Ricorrono in queste liriche, quasi a voler far da controcanto alla sangre derramada dell'amico caduto, il motivo dell'acqua per mezzo dei fiumi (Darro, Genil) così tanto cari a Federico.

C'è poi la dolente e celebre "Elegía a un poeta que no tuvo su muerte" scritta nel 1936, all'indomani del grave accaduto e poi inserita in Capital de la gloria, che reputo necessario riportare in forma integrale:

No tuviste tu muerte, la que a ti te tocaba. Malamente, a sabiendas, equivocó el camino. ¿Adónde vas? Gritando, por más que aligeraba no paré tu destino.

¡Que mi muerte madruga! ¡Levanta! Por las calles, los terrados y torres tiembla un presentimiento. A toda costa el río llama a los arrabales, advierte a toda costa la oscuridad al viento.

Yo, por las islas, preso, sin saber que tu muerte te olvidaba, dejando mano libre a la mía. ¡Dolor de haberte visto, dolor de verte como yo hubiera estado, si me correspondía!

Debiste de haber muerto si llevarte a tu gloria ese horror en los ojos de último fogonazo ante la propia sangre que dobló tu memoria, toda flor y clarísimo corazón sin balazo.

Mas si mi muerte ha muerto, quedándome la tuya, si acaso le esperaba más bella y larga vida, haré por merecerla, hasta que restituya a la tierra esa lumbre de cosecha cumplida.

In questo testo sembra quasi leggersi il rammarico per non aver potuto sostituire Federico in quel vile assassinio: «Malamente, a sabiendas, equivocó el camino", scrive. Non era quella la fine che dovevi fare, chiosa Alberti, con un linguaggio basico e piano, con il tormento per l'amico morto che gli invade i pensieri («iDolor de haberte visto, dolor, dolor!») e non gli dà scampo: non accetta il fatto che lui sopravviva mentre Federico se n'è andato per sempre: «Yo, por las islas, preso, si saber que tu muerte/te olvidaba, dejando mano libre 
a la mía». Lo smarrimento è tale che Alberti parla, invoca e cerca un tentativo di auto-annullamento («mi muerte ha muerto») e al contempo, quale testamento morale per l'amico, si assume l'obbligo di vivere la vita nel migliore dei modi, per celebrare degnamente e protrarre il ricordo: «si acaso le esperaba más bella y larga vida,/ haré por merecerla, hasta que restituya/ a la tierra esa lumbra de cosecha cumplida».

Durante la sua permanenza nel Lazio, Alberti raccolse un nutrito numero di liriche scritte nel borgo di Anticoli Corrado, sui Castelli Romani, che pubblicò nel 1972 sotto il titolo di Canciones del Alta Valle del Aniene. A distanza di trentasei anni dalla morte di García Lorca, Alberti dedica una nuova lirica dal titolo "Federico" dove, nella ritrovata calma che fa seguito al suo allontanamento da Roma e con l'immersione in uno spazio naturalistico e pacificato, il poeta di Cadice riesce a ritrovare l'amico Federico:

Federico.

Voy por la calle del Pinar para verte en la Residencia.

Llamo a la puerta de tu cuarto.

Tú no estás.

Federico.

Tú te reías como nadie.

Decías tú todas tus cosas

como ya nadie las dirá.

Voy a verte a la Residencia.

Tú no estás.

Federico.

Por estos montes del Aniene tus olivos trepando van.

Llamo a sus ramas con el aire.

Tú sí estás.

E, ancora, altri versi a lui dedicati negli anni successivi, rispettivamente le poesie dal titolo "F.G.L.", “Entre F.G.L. y R.A." contenute nella raccolta Fustigada luz 1972-1978 (1980) e “Me Ilaman de Granada”, “Por aquí Federico" e "Se me aparece la Alhambra" contenute, invece, in Versos sueltos de cada día (1982) a testimonianza di un rapporto continuo, imperituro, senza ostacoli che tracima ogni tempo. Segno evidente di un'amicizia vera e 
sentita umanamente, con lasofferenza devastante della perdita e al contempo la gioia dei momenti felici che risalgono alla mente. Alberti non solo ha mantenuto vivo l'amico Federico impegnandosi negli ultimi anni a collaborare a iniziative a lui dedicate, partecipando o contribuendo in maniera decisiva a nuove edizioni dell'opera del granadino, con grande amore e solidarietà, ma ha mantenuta viva la fiammella dentro di lui di quell'esperienza effervescente, arricchente e unica, seppur troppo breve, che fu Federico García Lorca. Così con l'immortalità che è carattere peculiare della poesia - Alberti è ancora lì ad attendere il suo amico, teso a cercare di recepire dall'ambiente i segnali della sua manifestazione, a intuire la sua presenza in ciò che lo circonda, per continuare quei discorsi, tra battute simpatiche e letture delle loro opere, da cui nacque questa amicizia che, da letteraria si è fatta storica e da storica, senza tempo: «Yo te sigo esperando. Hubiera preferido estarte yo esperando».

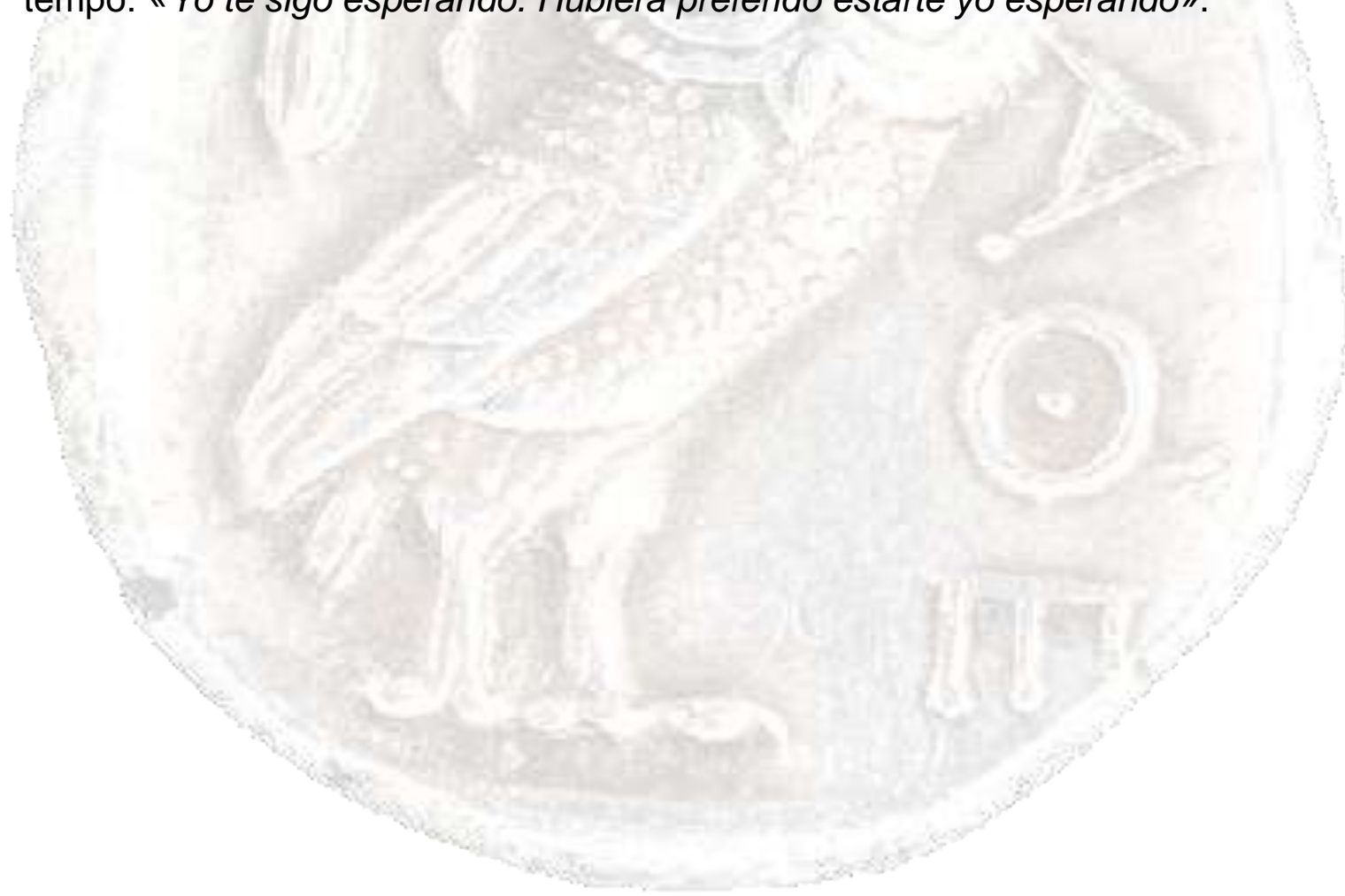




\section{Note}

1 Una generazione che, secondo Ignazio Delogu, si sfaldò negli anni successivi per una serie ampia di ragioni che vanno dall'accusa che il surrealismo e l'ultraismo (Guillermo de Torre, Vicente Huidobro) ne avessero minato la struttura interna (bisogna sempre ricordare che non tutti i poeti della generazione furono coinvolti da una fase surrealista), finanche da episodi cogenti come la morte indotta e violenta di alcuni (Lorca), l'allotanamento dalla scena di altri (Miguel Hernández imprigionato) che provocarono una vera dispersione e isolamento tra i membri che, in precedenza, raccolti attorno alla Residencia de Estudiantes di Madrid, avevano costituito un gruppo eterogeneo e abbastanza affiatato. Le posizioni ideoligiche di alcuni (Lorca e la dedizione agli emarginati) e la radicalizzazione comunista di altri (Alberti e il suo marxismo) probabilmente gravarono sull'interno del gruppo, dal momento che la generazione non si configurava a livello identitario e ideologico uniformemente con la sinistra. Secondo Delogu nel 1931 già si poteva considerare esaurita questa generazione che, appunto, "entra in una fase di contrasti che si manifestano con silenzi, reciproche indifferenze, esclusioni, discriminazioni che rispecchiano, [...] l'elemento nuovo e decisivo, lo scontro in atto nel paese. Si potrebbe dire che i conflitti interni e internazionali passano all'interno della Generazione stessa», in IGNAZIO DELOGU, Rafael Alberti, La Nuova Italia, Firenze, 1972, p. 92.

2 Interessanti indagini che consentono un approfondimento, in forma breve, attorno alla fase surrealista di Alberti sono "Buñel e la generazione del '27: appunti su forme comuni" di Maria Grazia Profeti e "In margine all'antologia. Per conoscere Rafael Alberti di Ignazio Delogu" di Marcella Eusebi Ciceri. La fase surrealista di Lorca, invece, venne studiata in Italia da Piero Menarini, docente emerito dell'università di Bologna, che pubblicò Poeta en Nueva York di Federico García Lorca. Lettura critica, La Nuova Italia, Firenze, 1975.

3 IGNAZIO DELOGU, Rafael Alberti, Op. Cit., p. 67. Tale concetto è reiterato poche pagine dopo: «Degli angeli esprime il momento di maggiore depressione di Alberti e dà sfogo alla componente pessimistica della sua personalità», in IGNAZIO DELOGU, Rafael Alberti, Op. Cit., p. 72.

4 MAIRA NEGRONI, Rafael Alberti: l'esilio italiano, Vita e Pensiero, Milano, 2001, p. 86.

5 Tra gli altri traduttori di Alberti figurano Dario Puccini e Marcella Eusebi Ciceri.

6 CARLO BO, "Alberti e Carrieri: entrambi figli di Apollinaire", Europeo, 6 dicembre 1964, pp. 104-106. 
7 MAIRA NEGRONI, Rafael Alberti: l'esilio italiano, Op. Cit.

8 IGNAZIO DELOGU, Per conoscere Rafael Alberti, Mondadori, Milano, 1977, p. XIV.

9 José Maria Pemán (1897-1981) fu uno degli uomini di spicco della stagione del regime di Miguel Primo de Rivera (1870-1930); rivestì incarichi importanti tra i quali Presidente dell'Ateneo Gaditano dal 1927, incarico che perdette di colpo con l'istaurazione della Repubblica. Nel 1931 Rafael Alberti, impegnato all'università di Cadice per una lettura poetica, lo inviò ad andarsene da lì perché la sua presenza non era assolutamente gradita. Pochi giorni dopo si sarebbe proclamata la Repubblica; Pemán nel 1933 entrò nel nascente partito Renovación Española, confluito poi in Acción Española, che avrebbe sostenuto la Falange negli anni a venire. Poeta e scrittore di regime poi, con una intensa attività letteraria tanto per la poesia, narrativa, critica, pubblicò il Romancero carlista, oltre alla composizione degli inni della CEDA, l'inno nazionale (Marcha Real), delle Forze Armate e altri. A Burgos, dove venne inviato, fu resposabile della sorveglianza, controllo e censura delle opere letterarie e di stampa, gestendo una massiccia opera di epurazione di dissidenti al regime. Nominato Ministro della Cultura sotto Franco, venne da tutti considerato "il genio del regime". Tra le maggiori opere figurano il Poema del la Bestia y el Ángel, per la narrativa Historia del fantasma y doña Juanita, Cuentos sin importancia, La novela de San Martín..., per il teatro coltivò il dramma storico-religioso in verso (El divino impaciente, Cuando las Cortes de Cádiz y Cisneros), la tematica andalusa (Noche de levante en calma) e la commedia di costume (Julieta y Romeo y El viento sobre la tierra).

10 Antonio Burgos, "Pemán y Alberti: las dos Españas de Cádiz", in Antología de Recuadros,

http://www.antonioburgos.com/antologia/archivo/diario16/ar012993.html

11 La prima edizione venne pubblicata dalla casa editrice Joaquin Mortiz in Messico. In Spagna il volume poté essere pubblicato solo nel 1974.

12 Maira Negroni sostiene a tal riguardo che "Pericolo, a cui accenna il titolo non è solo quello di rimanere vittime del traffico sregolato della capitale, ma si riferisce soprattutto all'intenso potere di seduzione che esercita la bellezza di Roma, capace di far vagabondare per piazze e vicoli chi vi si addentra e di assorbirlo completamente in essa, come al poeta stesso era accaduto", in MAIRA NEGRONI, Rafael Alberti: I'esilio italiano, Op. Cit., p. 38. È un'ipotesi senz'altro suggestiva che mi pare possa essere plausibile assieme al non meno rilevante pericolo cogente che l'io lirico fisicamente prova dinanzi al caos della città.

13 MAIRA NEGRONI, Rafael Alberti: l'esilio italiano, Op. Cit., p. 34. 
14 Si tratta di un «linguaggio indecoroso, spesso triviale e infarcito di termini volgari», in MAIRA NEGRONI, Rafael Alberti: l'esilio italiano, Op. Cit., p. 36.

15 «La città presenta un certo grado di fatiscenza e [..] alcune sue zone sono state molto trascurate dai suoi abitanti», in MAIRA NEGRONI, Rafael Alberti: l'esilio italiano, Op. Cit., p. 56.

16 Uno degli Once sonetos, una delle sezioni di questo libro, ha per oggetto proprio il sovrappopolamento felino, esso porta il titolo "Gatos, gatos y gatos...".

17 «L'atto dell'orinare viene associato con il fatto stesso dell'esser vivi», in MAIRA NEGRONI, Rafael Alberti: l'esilio italiano, Op. Cit., p. 43.

18 La prima edizione dell'opera, in lingua spagnola, uscì per la casa editrice Losada di Buenos Aires. Nello stesso anno uscì la prima versione tradotta in italiano, da Ignazio Delogu, con il titolo di Disprezzo e meraviglia per gli Editori Riuniti di Roma. II volume era dedicato al popolo vietnamita, in segno di solidarietà, nel periodo del conflitto con gli Usa. La prima edizione in territorio spagnolo è datata 1979 per i tipi di Seix Barral di Barcellona. La pubblicazione poté avvenire solo dopo il decesso del dittatore Francisco Franco.

19 MAIRA NEGRONI, Rafael Alberti: l'esilio italiano, Op. Cit., p. 67. 20 Ivi, p. 69.

21 Pedro Salinas ebbe a rivelare che in entrambi i poeti «nel loro verso, [si rivelavano] tracce di un'influenza del grande poeta nicaraguegno [Rubén Darío]", in PEDRO SALINAS, "Una antología de la poesía española contempóranea", Literatura española siglo XX, pp. 211-212.

22 Per quanto attiene all'unità e all'ordine di questa serie di sonetti dedicata da Alberti a Lorca si veda il saggio: HILARIO JIMÉNEZ GÓMEZ, "Alberti imagina a Lorca: los cuatros sonetos de Marinero en tierra", in Anuario de Estudios Filológcos, XXIV, 2001, pp. 243-253. Secondo quanto riportato da Maria Cristina Assumma nel suo saggio l'ultimo sonetto venne scartato da Alberti per l'edito princeps di Marinero en tierra, opera alla quale era destinata la serie. In altre edizioni il secondo sonetto appare in una diversa localizzazione.

23 Secondo Hilaro Jiménez Gómez il quarto sonetto non entrò a far parte di Marinero en tierra per volere dello stesso Alberti che, forse, lo riteneva non in linea agli altri tre componimenti, dato che vi era presente una simbologia leggibile a livello erotico («Clava tu espada en mí, tú, pez-espada»), in HILARO JIMENEZ GÓMEZ, "Alberti imagina a Lorca: los cuatro sonetos de Marinero en tierra", Op. Cit. 
24 Poesia "Primavera".

25 Poesia "Otoño".

26 Poesia "Invierno".

27 Ibidem.

28 Poesia "Verano".

29 Nella poesia “Invierno" Rafael Alberti si dipinge, umiliandosi, «débil junco frío».

30 RAFAEL ALBERTI, Degli angeli, Einaudi, Torino, 1966, pp. 5-6.

31 IGNAZIO DELOGU, Per conoscere Alberti, Op. Cit., p. LXII.

32 Lorca, a cui spettò questa inumana fine, aveva posto il tema della morte quale uno degli aspetti centrali e piu ricorrenti della sua intera produzione poetica e non solo. Anche nelle opere teatrali, infatti, l'autore aveva messo in risalto la dimensione tragica e ineluttabile di un destino umano improntato alla discordia, allo scontro, alla mancanza di integrità morale. In relazione alla tematica della morte, così presente in Lorca da divenire immancabile, Alberti ebbe ad osservare: «Anch'io mi pongo il problema della morte, anche se nella mia poesia non costituisce un problema centrale, come lo era, per esempio, in quella di Lorca. Ma sin quando il problema non sia risolto, nel senso che restano ancora delle domande senza risposta, credo che si abbia il diritto di porselo», in IGNAZIO DELOGU, Per conoscere Rafael Alberti, Op. Cit., p. LVII.

33 A questi aspetti ho dedicato un precedente saggio intitolato "Un nardo reciso. Le ultime ore di vita di Federico e il lutto della poesia" in A. MELILLO, G. MICHELI, La memoria, Ladolfi, Borgomanero, 2016. In esso si fa riferimento, tra le altre cose, alla partecipazione di Lorca - mediante la sottoscrizione di manifesti - ad alcune battaglie popolari nella forma della denuncia ad alcune dittature, come il salazarismo nel vicino Portogallo e si parla del progetto di-teatro itinerante La Barraca, molto seguito e particolarmente efficace, che secondo molti rappresentò la goccia che fece traboccare l'acqua dal vaso. Dal momento che venne accusato di adoperare questo teatro all'aire libre per fare proselitismo marxista. Maria Cristina Assumma, nelle note a fine capitolo del suo saggio, tiene, però, ad osservare: «Alla vigilia delle elezioni politiche del febbraio del '36, Lorca non esita a sottoscrivere il manifesto e ad aderire alle iniziative che un gruppo di intellettuali, capeggiati da Rafael Alberti e da María Teresa León, promuovono in favore del Frente Popular. [...] Lorca partecipa, insieme al "poeta revolucionario" e sua moglie, a una manifestazione di solidarietà con il leader comunista 
brasiliano Luis Carlos Prestes, arrestato dal dittatore Getulio Vargas con altri lavoratori», in MARIA CRISTINA ASSUMMA, Lorca e Alberti. Tradizione e avanguardia, Op. Cit., p. 18.

34 Ignazio Delogu osserva «che in García Lorca vi fosse una componente di sinistra è fuor di luogo», in IGNAZIO DELOGU,

Rafael Alberti, Op. Cit., p. 90.

35 ॥l vero titolo dell'opera è "Con los zapatos puestos tengo que morir". Alberti ricorda la genesi di questo testo con il quale inaugurò la sua "discesa in campo" e la fase poetica dell'impegno civile: "Con una desesperación borrosa que me llevaba morder el suelo, este poema que subtitulé Elegía Cívica señala mi incorporación a un universo nuevo, por el que entraba a tientas, sin preocuparme siquiera adónde me conducíia", il RAFAEL ALBERTI, La Arboleda Perdida, 1975, p. 290.

36 IGNAZIO DELOGU, Per conoscere Rafael Alberti, Op. cit.

37 Nell'occasione del centenario della nascita di Alberti, celebrata a Granada nel 2002, sua figlia Aitana rivelò che la brutale morte di García Lorca originò in suo padre «un sentimiento de culpa que arrastró durante toda su vida», in MARIA CRISTINA ASSUMMA, Lorca e Alberti, Op. Cit., p. 81.

38 Vittorio Bodini osservò «Alberti è il poeta spagnolo che sia sceso in piazza, accanto agli studenti che protestarono contro la dittatura di Primo de Rivera con manifestazioni represse nel sangue », in RAFAEL ALBERTI, Degli Angeli, Einaudi, Torino, 1966, p. 11.

39 MARIA CRISTINA ASSUMMA, Lorca e Alberti, Op. Cit., p. 14.

40 Tra le altre opere di poesia surrealista prodotte dagli altri esponenti della generazione del '27 figurano Ámbito, Espadas como labios e La destrucción o el amor di Vicente Aleixandre, Un río, un amor di Luís Cernuda, La voz cautiva di Emilio Prados.

41 Rafael Alberti, "Su Poeta en Nueva York di Federico García Lorca", Sur, Buenos Aires, dicembre 1960. II testo è stato edito in versione tradotta in italiano in IGNAZIO DELOGU, Per conoscere Rafael Alberti, Op. Cit., dal quale si cita.

42 MARIA CRISTINA ASSUMMA, Lorca e Alberti. Op. Cit., p. 98.

43 Ivi, p. 100.

44 Ivi, p. 13. 
45

IGNAZIO DELOGU, Per conoscere Rafael Alberti, Op. Cit.

46 FEDERICO GARCÍA LORCA, Tutte le poesie e tutto il teatro, traduzioni a cura di Claudio Rendina ed Elena Clementelli, Newton Compton, Roma, 2009, p. 692.

47 Ivi, p. 679.

48 Ivi, p. 684.

49 Ivi, p. 686.

50 Ivi, p. 684.

51 RAFAEL ALBERTI, Cal y canto, Seix Barral, Barcelona, 1929.

52 AA.VV., Antología comentada de la Generación del 27, introducción de Víctor García de la Concha, guía de lectura de Juan Francisco Peña, Espasa Calpe, Madrid, 2007, p. 314.

53 Comune fu pure il fascino nei confronti del mondo taurino; entrambi i poeti dedicarono delle elegie al compianto Ignacio Sánchez Mejías (1891-1934), torero, poeta e anfitrione messicano che nel 1934 nella plaza de toros di Manzanares venne incornato a morte dal toro "Granadino": Lorca il celebre Llanto por Ignacio Sánchez Mejías e Alberti Verte y no verte, entrambi pubblicati nel 1935. Riferimenti alla corrida, alle movenze del torero, ai suoi tercios sono presenti in varie poesie e nelle opere teatrali di entrambi i poeti.

54 Buster (vero nome: Joseph Frank) Keaton (1895-1966) fu un attore, regista e sceneggiatore americano tra i principali del cinema muto. Attore sin da giovanissimo, in teatro recitò la parte del bambino de /l piccolo Lord, nel 1919 fondò una compagnia propria, la Buster Keaton Comedies con la quale, dal 1920 al 1923, mise in piedi almeno una ventina di cortometraggi dei quali curò la regia. Tra le tematiche di fondo, il doppio, il rapporto tra veglia e sonno, tra realtà e finzione, l'importanza dell'imprevisto. Con l'arrivo del sonoro iniziarono i problemi per Keaton che, non possedendo di una voce idonea a questo genere di presenze in fatto di attore, decreto una sorta di declino, la cui angoscia in termini personali si accrebbe anche a seguito dell'uso di alcool. A tutto ciò si aggiunsero anche problemi familiari, tra divorzi, allontanamenti dei figli e ingiunzioni di pagamento. La sua situazione sembrò risollevarsi negli anni '30-'40 durante i quali riuscì a riottenere delle commesse e per vari cortometraggi. Nel 1960 il cinema, riconoscendo il suo contributo artistico, gli diede l'Oscar alla Carriera. Come si legge nella relativa pagina Wikipedia, «il suo cinema fu un meccanismo con continui rovesciamenti di senso, all'insegna di un esercizio continuo della logica: gli oggetti che cambiano di senso, le azioni semplici diventano complesse 
e quelle impossibili diventano facilissime, ciò che sembra innocuo diventa un pericolo e le avversità si rivelano aiuti impensati». In questa definizione sembra ben riassulto l'approccio surreale e fantasmagorico di Keaton che ben lo collega a Lorca-Alberti, poeti affascinati da questo mondo cinematografico muto denso di mistero, equivoci e polisenso.

55 Tutte le citazioni da quest'opera, in versione tradotta in italiano, sono tratte da GARCÍA LORCA FEDERICO, Tutte le poesie e tutto il teatro, traduzioni a cura di Claudio Rendina ed Elena Clementelli, Newton Compton, Roma, 2009, pp. 10871088.

56 MARIA CRISTINA ASSUMMA, Lorca e Alberti, Op. Cit., p. 50.

57 Ivi, p. 50. In merito alla sua lettura dei contenuti il critico ha osservato: «Buster Keaton è in realtà un assassino che però non sa di essere tale, perché si è dimenticato di aver compiuto la strage dei propri figli, avengola eseguita con indifferenza», in MARIA CRISTINA ASSUMMA, Lorca e Alberti, Op. Cit., p. 53.

58 IGNAZIO DELOGU, Rafael Alberti, Op. Cit., pp. 96-97.

59 Si tratta del testo pronunciato alla conferenza tenutasi a Buenos Aires nel 1941, successivamente pubblicato insieme alla sua opera "Romance di Fermín Galán". La versione tradotta in italiano è inserita nel volume IGNAZIO DELOGU, Per conoscere Rafael Alberti, Op. Cit., dalla quale si cita.

60 Sono le "due Spagne" che ben dipinge anche García Lorca in Mariana Pineda ovvero la monarchia contro i moniti repubblicani da quest'ultima intesi come episodi di sovversismo e dunque punibili, anche con la morte, come è il caso della stessa Mariana. Fazioni che si ritrovano anche in Bodas de sangre dove due famiglie storicamente antagoniste si sfideranno, nella circostanza di un matrimonio, in un duello all'ultimo sangue teso a vendicare le morti precedentemente accadute e in difesa dell'onore familiare. Le ritroviamo anche in La casa de Bernarda Alba dove il dominio schizofrenico e dittatoriale di Bernarda Alba (un vero despota, immagine dell nascente classe franchista) si scontra duramente, con l'uso della violenza, con chi professa la democrazia e cerca di difendere il diritto alla libertà (la giovane Adela).

61 IGNAZIO DELGOU, Per conoscere Rafael Alberti, Op. Cit.

62 Ibidem

63 Si rifersce alla Regina Vittoria Eugenia di Battenberg (1887-1969), Regina di Spagna, consorte di Re Alfonso XIII. 
64 Si tratta del Cardinale Pedro Segura y Sáenz (1880-1957), arcivescovo di Toledo, arcivescovo di Siviglia e primate di Spagna. II cardinale Segura non mancò di esprimere pubblicamente e in più circostanze la propria opposizione al nuovo organo istituzionale venutosi a creare in Spagna, la Repubblica, dimostrando sempre appoggio a re Alfonso XIII.

65 Si riferisce al Principe Alfonso di Borbone-Spagna (1907-1938) allora Principe delle Asturie perché erede diretto del padre, il Re Alfonso XIII. Tuttavia, con l'avvento della Repubblica e della dittatura poi, il Principe Alfonso non divenne mai Re di Spagna mentre avrebbe dato i natali a Juan Carlos I che, nel 1978, secondo le volontà del dittatore Francisco Franco venuto a mancare nel 1975, venne incoronato $\mathrm{Re}$ di Spagna. II Principe Alfonso rimase così noto con l'appellativo di Conte di Barcellona.

66 L'opera andava in scena in un momento ancora abbastanza turbolento e non chiaro, appena un mese e mezzo dopo la proclamazione della Repubblica.

67 Cit. in ANTONINA RODRIGO, Margarita Xirgu, Flor del viento ediciones, Barcelona, 2005, p. 203. 


\section{Bibliografia}

AA.VV., Antología comentada de la Generación del 27, introducción de Víctor García de la Concha, guía de lectura de Juan Francisco Peña, Espasa Calpe, Madrid, 2007.

AA.VV., I poeti surrealisti spagnoli. Saggio introduttivo e antologia, a cura di Vittorio Bodini, Einaudi, Torino, 1963. ALBERTI RAFAEL, Degli angeli, traduzione di Vittorio Bodini, Einaudi, Torino, 1966.

ALBERTI RAFAEL, Federico García Lorca. Poeta y amigo, a cura di Luis García Montero, Editoriales Andaluzas Unidas, Granada, 1984.

ASSUMMA MARIA CRISTINA, Lorca e Alberti. Tradizione e avanguardia, Artemide, Roma, 2009. DELOGU IGNAZIO, Per conoscere Rafael Alberti, Mondadori, Milano, 1977.

DELOGU IGNAZIO, Rafael Alberti, La Nuova Italia, Firenze, 1973.

GARCÍA LORCA FEDERICO, Tutte le poesie e tutto il teatro, traduzioni a cura di Claudio Rendina ed Elena Clementelli, Newton Compton, Roma, 2009.

NEGRONI MAIRA, Rafael Alberti: I'esilio italiano, Vita e Pensiero, Milano, 2001. RODRIGO ANTONINA, Margarita Xirgu, Flor del viento ediciones, Barcelona, 2005.

SPURIO LORENZO, "Un nardo reciso. La morte di Federico e il lutto della poesia", in A. MELILLO, G. MICHELI, La memoria, Ladolfi, Borgomanero, 2016.

TEJADA, J.L., Rafael Alberti, entre la tradición y la vanguardia, Gredos, Madrid, 1977. 